\title{
Diagnostic accuracy of hysterosalpingography and laparoscopy in tubal patency
}

\author{
Palvi Banotra ${ }^{1 *}$, Zahoor Ahmad ${ }^{2}$
}

\author{
${ }^{1}$ Department of Health and Medical Education, Jammu, Jammu and Kashmir, India \\ ${ }^{2}$ Department of Statistics, University of Kashmir, Srinagar, Jammu and Kashmir, India
}

Received: 26 December 2021

Accepted: 10 January 2022

\section{*Correspondence: \\ Dr. Palvi Banotra, \\ E-mail: palvibntr@gmail.com}

Copyright: (c) the author(s), publisher and licensee Medip Academy. This is an open-access article distributed under the terms of the Creative Commons Attribution Non-Commercial License, which permits unrestricted non-commercial use, distribution, and reproduction in any medium, provided the original work is properly cited.

\begin{abstract}
Background: There is no doubt that health care providers face a great deal of challenge to deal with the infertility issue, a serious and tragic gynecological problem encountered in developing countries. Laparoscopy is considered as the gold standard for assessment of tubal factors of infertility, although because of its invasive nature, cost and the need for anaesthesia and hospitalization, HSG seems to be a basic routine procedure for tubal factors. Hence, the present study was designed to compare the accuracy of HSG with SSG for evaluation of tubal factor infertility. Laparoscopy is considered as the gold standard for assessment of tubal factors of infertility, although because of its invasive nature, cost and the need for anaesthesia and hospitalization, HSG seems to be a basic routine procedure for tubal factors. Currently with the availability of the ultrasonography machines with very good resolution, SSG can be simultaneously practiced with ultrasonography during day 7-9 of the menstrual cycle to assess tubal patency. Hence, the present study was designed to compare the accuracy of HSG with laparoscopyfor evaluation of tubal factor infertility.

Methods: The present prospective study on the diagnostic accuracy of hysterosalpingography (HSG) in the evaluation of infertility due to tubal patency has been conducted at Lala-Ded Hospital, Srinagar from September 2015 to February 2016. All the patients with infertility having regular menses, normal male partner with semen analysis and aging 20-30 years old have been included in the study. However, females who underwent HSG previously, case of unovulation despite clomiphene citrate, acute PID, salpingectomy, endometriosis, past history of oophorectomy and women beyond 35 years of age have been excluded in this study.

Results: The average age of patients was observed as $(26.7 \pm 4.81)$ years with majority of patients belonging to (25-29) year age group. We performed sensitivity analysis of HSG procedure by taking laparoscopic findings as gold standard; evidently, we found the sensitivity and specificity of HSG as $91.3 \%$ and $88.9 \%$ respectively. Interestingly, the diagnostic accuracy of HSG was observed as $89.7 \%$.

Conclusions: We found that HSG has satisfactory predictive value in identifying the factor of infertility. Laparoscopy is still a gold standard but if the purpose is attained with minimally invasive and cost-effective procedure having a good accuracy rate then over diagnosis should be avoided.
\end{abstract}

Keywords: Tubal patency, HSG, Laparoscopy

\section{INTRODUCTION}

There is no doubt that health care providers face a great deal of challenge to deal with the infertility issue, a serious and tragic gynecological problem encountered in developing countries. There are multifarious factors that are responsible for its rising trend. However, it has been seen that tubal occlusion is one of the most frequent causes of infertility in women. ${ }^{1,2}$ It has been observed by that tubal peritoneal factors are responsible for $35 \%$ to $40 \%$ of reproductive couples. ${ }^{3,4}$ Therefore the evaluation of tubal patency is necessary to plan the precise treatment protocol for the management of infertility. Laparoscopy and HSG are two most commonly exploited procedures used in the 
assessment of morphological exploration of fallopian tubes in developing countries. But sometimes due to lack of funds gynecologists have no choice but to prescribe only HSG. The HSG is a contrast enhanced fluoroscopic and flat plate study used to evaluate the endometrial cavity and fallopian tubes. It has been a test in the workup of infertile couples as a minimally invasive method of evaluating tubal patency and is performed as the first line approach for assessing tubal pathology. While as laparoscopy is considered the clinical reference test for diagnosing tubal pathology. ${ }^{5}$ Laparoscopy allows visualization of periadnexal adhesions and the presence of endometriosis, which cannot be done with HSG. ${ }^{6}$ However, the likelihood that a laparoscopy will show tubal occlusion after a normal HSG is very low. The present study has been conducted to evaluate the diagnostic accuracy of HSG in the detection of tubal patency and pelvic adhesion and compared it with laparoscopic investigations

\section{METHODS}

The present prospective study on the diagnostic accuracy of HSG in the evaluation of infertility due to tubal patency has been conducted at Lala-Ded hospital, Srinagar from September 2015 to February 2016. All the patients with infertility having regular menses, normal male partner with semen analysis and aging 20-30 years old have been included in the study. However, females who underwent HSG previously, case of anovulation despite clomiphene citrate, acute PID, salpingectomy, endometriosis, past history of oophorectomy and women beyond 35 years of age have been excluded in this study. Initially on the basis of inclusion and exclusion criteria, a random sample of 82 patients were included in the study, however, 14 patients conceived spontaneously and they were excluded and hence 68 patients remained in the study. After patients understood the mechanism of HSG and laparoscopy, they were assessed with both HSG and laparoscopy in a standard manner and chromo-pertubation test was done to confirm finding of HSG. All HSG's were performed in outpatient clinic of department of radiology between $7^{\text {th }}$ to $10^{\text {th }}$ days of menstrual cycle. Tubal-occlusions (one-sided tubal occlusions or two-sided tubal occlusions) were taken up as a single entity. Additional abnormalities of the uterine cavity were recorded as well. Tubal occlusions detected at HSG were compared with occlusions detected at laparoscopy. Sensitivity, specificity, positive predictive value, negative predictive value of HSG in the diagnosis of tubal occlusion was calculated regarding laparoscopy as the reference standard.

\section{Statistical methods}

The recorded data was compiled and entered in a spreadsheet (Microsoft excel) and then exported to data editor of SPSS version 20.0 (SPSS Inc., Chicago, Illinois, USA). Statistical software SPSS (version 20.0) and Microsoft excel were used to carry out the statistical analysis of data. Continuous variables were expressed as Mean \pm SD and categorical variables were summarized as percentages. Diagnostic accuracy (Sensitivity, Specificity, PPV and NPV) of HSG in diagnosis of infertility was obtained taking laparoscopy as gold standard.

\section{RESULTS}

In this section we will present the results obtained in the study in tabular form.

We observe that the average age of patients was (26.7 \pm 4.81$)$ years with majority of patients belonging to (25-29) year age group. The minimum and maximum age of studied patients was observed as 20 years and 33 years respectively instead of was reported a 11.8 years and 61.8 years.

Table 1: Age distribution of study patients.

\begin{tabular}{|lll|}
\hline Age (years) & Number & Percentage $(\%)$ \\
\hline $\mathbf{2 0 - 2 4}$ & 18 & 26.5 \\
\hline $\mathbf{2 5 - 2 9}$ & 42 & 61.8 \\
\hline $\mathbf{3 0 - 3 4}$ & 8 & 11.8 \\
\hline Total & 68 & 100 \\
\hline Mean \pm SD & (Range) $=\mathbf{2 6 . 7} \pm \mathbf{4 . 8 1}(\mathbf{2 0 - 3 3 )}$ \\
\hline
\end{tabular}

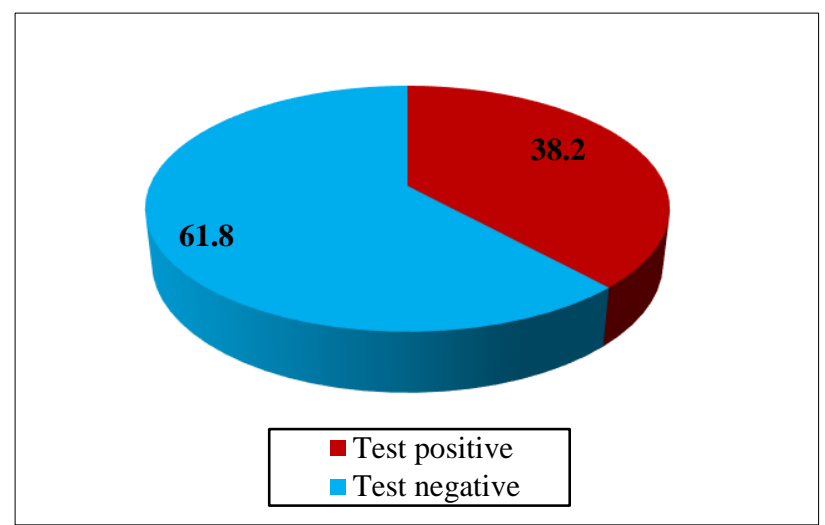

Figure 1: HSG findings of study patients.

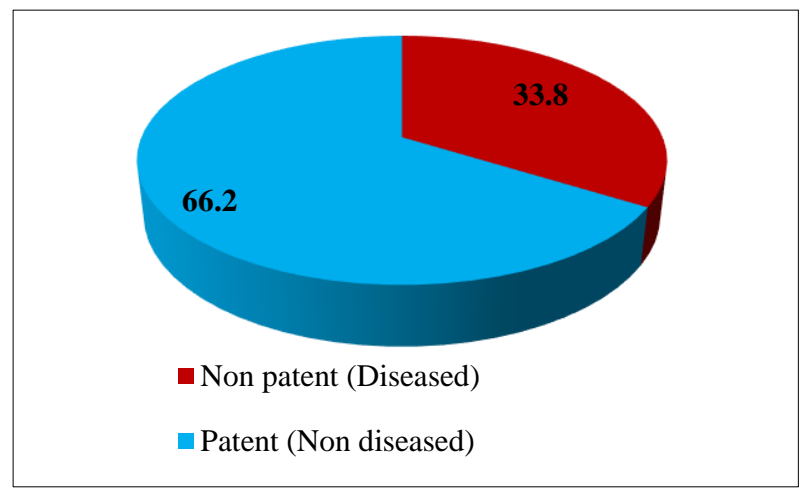

Figure 2: Laparoscopy findings of study patients.

We assessed patients with the help of HSG X-ray test and evidently $38.2 \%$ were tested positive and 61.8 were tested negative. 
With the help of laparoscopic investigation, we observe that $33.8 \%$ had tubal blockage and $66.2 \%$ had patent tubes.

Table 2: Comparative findings of HSG and laparoscopy.

\begin{tabular}{|c|c|c|c|}
\hline \multirow[b]{2}{*}{ HSG } & \multicolumn{2}{|c|}{ Laparoscopy } & \multirow[b]{2}{*}{ Total } \\
\hline & $\begin{array}{l}\text { Non patent } \\
\text { (diseased) }\end{array}$ & $\begin{array}{l}\text { Patent (non- } \\
\text { diseased) }\end{array}$ & \\
\hline Test positive & 21 & 5 & 26 \\
\hline Test negative & 2 & 40 & 42 \\
\hline Total & 23 & 45 & 68 \\
\hline
\end{tabular}

We made a comparative analysis of HSG and laparoscopic investigation and observed that out of 23 non patent (tubal blockages) reflected by laparoscopy, 21 of them were shown positive by HSG procedure and only two patients had a negative HSG study. Likewise, out of 45 patent laparoscopic findings, of them HSG showed 40 are negative and only 5 patients had positive HSG finding.

Table 3: Diagnostic accuracy of HSG in diagnosis of infertility taking laparoscopy as gold standard.

\begin{tabular}{|lll|}
\hline Variables & Values & $\mathbf{9 5} \%$ CI \\
\hline Sensitivity & 91.3 & $73.20-97.58$ \\
\hline Specificity & 88.9 & $76.51-95.16$ \\
\hline PPV & 80.8 & $62.12-91.49$ \\
\hline NPV & 95.3 & $84.21-98.68$ \\
\hline Diagnostic accuracy & 89.7 & $80.24-94.92$ \\
\hline
\end{tabular}

We performed sensitivity analysis of HSG procedure and observed that it has sensitivity of around $91.3 \%$ and $88.9 \%$ specificity. Interestingly, the diagnostic accuracy of HSG was observed as $89.7 \%$

\section{DISCUSSION}

In the present study, we comprehensively analyzed the role of HSG and laparoscopy in the evaluation of infertility. We observed the average age of patients as $(26.7 \pm 4.81)$ years and majority of infertile patients were belonging to (25-29) age group with minimum and maximum age of studied patients was respectively recorded as 11.8 years and 61.8 years. Farideh et al in a likewise study reported that mean age of such patients is $29.3 \pm 62.5$ which is almost similar to our observation. ${ }^{7}$ We made a comparative analysis of HSG and laparoscopic investigation and observed that out of 23 non patent (tubal blockages) reflected by laparoscopy, 21 of them were shown positive by HSG procedure and only two patients had a negative HSG study. Likewise, out of 45 patent laparoscopic findings, of them HSG showed 40 are negative and only 5 patients had positive HSG finding. Interestingly out of all 26 cases of positive HSG results, 21 patients were shown non-patent by laparoscopic investigation which means an agreement in $(80.76 \%)$ cases. Similarly, out of 42 HSG negative results, 40 cases were shown non-patent by laparoscopy which means good agreement. The diagnostic precision of HSG was reported by a good corpus of scholars. For instance, authors like Fayaz et al reported that out of 420 patients, it was demonstrated that HSG is as good as laparoscopy in the detection of tubal patency and associated obstruction; howbeit, they did not rule out the superiority of laparoscopy in the evaluation of peritubal adhesions and other associated pathologies. ${ }^{8}$ The potential of laparoscopy over the use of HSG cannot be ignored but sometimes due to lack of funds, inadequate health facilities, noninvasive and cost effectiveness, gynecologists have no choice but to prescribe only HSG. In the present study, we performed sensitivity analysis of HSG procedure and observed that it has a sensitivity of around $91.3 \%$ and $88.9 \%$ specificity. Interestingly, the diagnostic accuracy of HSG was observed as $89.7 \%$. However, the false positive and false negative rate of HSG for diagnosing tubal patency was respectively observed as $8.69 \%$ and $12.5 \%$. These results are contemporary to the results reported by numerous authors, for instance; Goreekhanloo et al reported the sensitivity and specificity of HSG in detection of bilateral occlusion are $89.5 \%$ and $90 \%$ respectively, which is in agreement with our results. In a study due to Jain et al it was found that the sensitivity of HSG is around $93.33 \%$ and specificity was $91.11 \%{ }^{9}$ However Tvarijonaviciene et al reported that the sensitivity, specificity, and accuracy of HSG for the detection of unilateral and bilateral tubal occlusion were $75 \%, 91.2 \%, 89.5 \%, 75 \%, 97.2 \%$, and $96.6 \%$, respectively. ${ }^{10}$ Comparative analysis of (HSG vs laparoscopy) on a patient population of 102 infertile women was performed by Vasiljevic et al and it was demonstrated that the HSG and laparoscopy are yielding almost same results on unilateral and bilateral tubal blockages in $61.5 \%$ and $70.4 \%$ cases respectively. ${ }^{11} \mathrm{We}$ observed the diagnostic accuracy, negative predictive value and the positive predictive value of HSG in the present study as $89.7 \%, 95.3 \%$ and $80.8 \%$ respectively. In a likewise study by Jain et al the positive predictive value and negative predictive value was respectively reported as is $(77.77 \%)$ is $(97.61 \%)$ which is almost similar to our observations. The present study demonstrated the high diagnostic value and consistency HSG in tubal patency compared with laparoscopy. Therefore, HSG must be used as a first tool for the assessment of underlying reasons that leads to infertility. However, for highly suspicious results laparoscopy can be exploited to minutely observe the subtle differences.

HSG is useful in making decisions regarding further procedures for the diagnosis of infertility and should still serve as a useful primary investigation. HSG and laparoscopy are not alternative, but are the complementary methods in the examination of tubal blockage and since in the present study, the patient population was small therefore there is scope to conduct a study on larger sample size.

\section{CONCLUSION}

The present study revealed a good sensitivity, specificity and an impressive diagnostic value in the evaluation of 
infertility due to tubal blockages. We found that HSG has satisfactory predictive value in identifying the factor of infertility. Laparoscopy is still a gold standard but if the purpose is attained with minimally invasive and costeffective procedure having a good accuracy rate then over diagnosis should be avoided

Funding: No funding sources Conflict of interest: None declared

Ethical approval: The study was approved by the Institutional Ethics Committee

\section{REFERENCES}

1. Adamson PC, Krupp K, Freeman AH, Klausner JD, Reingold AL, Madhivanan P. Prevalence \& correlates of primary infertility among young women in Mysore, India. Indian J Med Res. 2011;134:440-6.

2. Sami N, Ali TS, Wasim S, Saleem S. Risk factors for secondary infertility among women in Karachi, Pakistan. PLoS One. 2012;7(4):e35828.

3. Lindsay TJ, Vitrikas KR. Evaluation and treatment of infertility. Am Fam Phys. 2015;91:308-14.

4. Otta SP, Reddy RG, Sangvikar S, Tripathy R. Ayurvedic management of female infertility due to tubal blockage. J Complement Integr Med. 2021.

5. Robabeh M, Roozbeh T. Comparison of hysterosalpingography and laparoscopy in infertile Iranian women with tubal factor. Ginekol Pol. 2012;83(11):841-3.
6. Sakar MN, Gul T, Atay AE, Celik Y. Comparison of hysterosalpingography and laparoscopy in the evaluation of infertile women. Saudi Med J. 2008;29(9):1315-8.

7. Farideh G, Rastegar F. Comparison of hysterosalpingography and laparoscopy in evaluation of female infertility. Med Res Arch. 2017;5(6):1-12.

8. Fayez JA, Mutie G, Schneider PJ. The diagnostic value of hysterosalpingography and laparoscopy in infertility investigation. Int $\mathrm{J}$ Ferti. 1988;33(2):98-101.

9. Jain P, Bansal D, Deodhar P. Reemerging role of HSG Vs laparoscopy for infertility work- up at rural hospital set up. J Res Med Den Sci. 2015;3(4):287-9.

10. Tvarijonaviciene E, Nadisauskiene RJ. The value of hysterosalpingography in the diagnosis of tubal pathology among infertile patients. Medicina (Kaunas). 2008;44(6):439-48.

11. Vasiljevic M, Ganovic R, Jovanovic R, Markovic A. Diagnostic value of hysterosalpingography and laparoscopy in infertile women. Srp Arh Celok Lek. 1996;124(5-6):135-8

Cite this article as: Banotra $\mathrm{P}, \mathrm{Ahmad} \mathrm{Z}$. Diagnostic accuracy of hysterosalpingography and laparoscopy in tubal patency. Int J Reprod Contracept Obstet Gynecol 2022;11:543-6. 